\title{
Sequential Process Optimisation Using Genetic Algorithms
}

\author{
V. Oduguwa, A. Tiwari and R. Roy \\ Enterprise Integration, \\ School of Industrial and Manufacturing Science, \\ Cranfield University, Cranfield, Bedford, \\ MK43 0AL, United Kingdom (UK). \\ voduguwa@hotmail.com, \{a.tiwari,r.roy\}@cranfield.ac.uk
}

\begin{abstract}
Locating good design solutions within a sequential process environment is necessary to improve the quality and overall productivity of the processes. Multi-objective, multi-stage sequential process design is a complex problem involving large number of design variables and sequential relationship between any two stages. The aim of this paper is to propose a novel framework to handle real-life sequential process optimisation problems using a Genetic Algorithm (GA) based technique. The research validates the proposed GA based framework using a real-life case study of optimising the multi-pass rolling system design. The framework identifies a number of near optimal designs of the rolling system.
\end{abstract}

\section{Introduction}

Process optimisation involves the generation of optimal design solutions for individual units of the process including both design information such as the geometrical size of a unit and the operating conditions for the unit which are used to meet the desired reqirements of the overall process. Process optimisation problems are complex and can be charateritised as having multiple stages. Individual units of the process systems are connected to form multiple stages of the overall process problem. The nature of this association could be sequential or non-sequential. Sequential nature of the process offers the separate subsystems a dependency link where the output relationship of a subsystyem becomes the input relationship of the subsequent subsystem in an orderly manner. Non-sequential on the other hand is nonordered association. This paper aims to propose a novel optimisation framework for handling sequential process optimisation problems.

Traditional methods of solving the sequential process optimisation problems are based on trial and error. This relies on manually adopting existing designs to produce the required design where a large set of design variables are changed, one variable at a time. Since the human can only deal with up to 5-10 variables at any single time [1], the optimisation task becomes slow and often results to sub optimum solutions.

A review of the literature reveals that there is very little work based on optimisation approaches reported for dealing with sequential process optimisation problems. However, there are some related works reported in metal forming in general. Several 
authors have adopted the finite element (FE) based method since it provides detailed information about the domain being studied. Kobayashi [2] applied a FE based backward tracing technique to design a pre-form in a shell housing. This was also applied to plane-strain rolling problems [3] and disk forging. This technique has been shown to discover the desired final shape in various forming problems. However, backward tracing technique, when used alone, cannot uniquely determine the optimal solutions due to the presence of diverse and multiple loading solution paths. It tends to be more efficient when the loading path is known, however this can lead to difficulties in those problems where the search space is unknown especially when multiple diverse loading paths are present.

Several authors have also used derivative based approaches to solve sequential process optimisation problems. Joun and Hwang [4] developed a FE based process optimisation technique and applied it to a die profile design extrusion problem. In spite of reported successes, the derivative based approaches require an initial guess, which can influence the search. They also tend to get stuck in sub-optimal solutions and an algorithm that is efficient in solving one optimisation problem may not be efficient in solving a different optimisation problem [5].

In order to address these limitations, a number of authors [6] are now adopting genetic algorithms (GAs) with embedded FE solver to automate the search for good quality solutions. GAs are adaptive methods used to solve search and optimisation problems, based on genetic processes of biological organisms [7]. Roy et al. [8] implemented an adaptive Micro Genetic Algorithm for shape optimisation of process variables in multi-pass wire drawing processes. Hwang and Chung [9] proposed a modified micro genetic algorithm for the optimisation of die shape in extrusion. These GA based techniques provide an algorithmic framework to deal with the parameter optimisation in sequential process optimisation problems. The techniques can deliver multiple good solutions, which speed up the design process. However, the GA based approach using the FE solver as embedded optimiser incurs severe computational cost in real-life problems. This problem intensifies since GA techniques require large number of solutions for convergence. This inhibits the use of algorithmic approaches to real-life sequential process optimisation. Therefore, this paper applies the proposed framework for the multi-objective design optimisation of a four pass ovalround rolling pass using mathematical process model developed from rolling theory.

\section{Proposed Optimisation Framework}

This section proposes a novel optimisation framework for handling real-life sequential optimisation problems. The solution strategy adopted by the optimisation framework is based on the GA. The dependency link between stages is modelled based on informing the subsequent stage $(i)$ of the move made by the (i-1)th stage such that the solution alternatives considered for the ith stage take into account the move made by the previous stage.

The GA based approach was selected because they have been used extensively as search and optimisation tools in various problem domains and the primary reasons for their success are applicability, ease of use and global perspectives. NSGA II was 
selected as the most popular multi-objective GA algorithm for the optimisation [5]. The following section presents the optimisation framework for real-life sequential optimisation problems. Figure 1 shows a flow chart of the solution technique. The solution algorithm consists of three main parts: the NSGA-II algorithm, the multistage model and the multi-objective fitness assignment. A brief description of the model and the coding scheme adopted is given below.

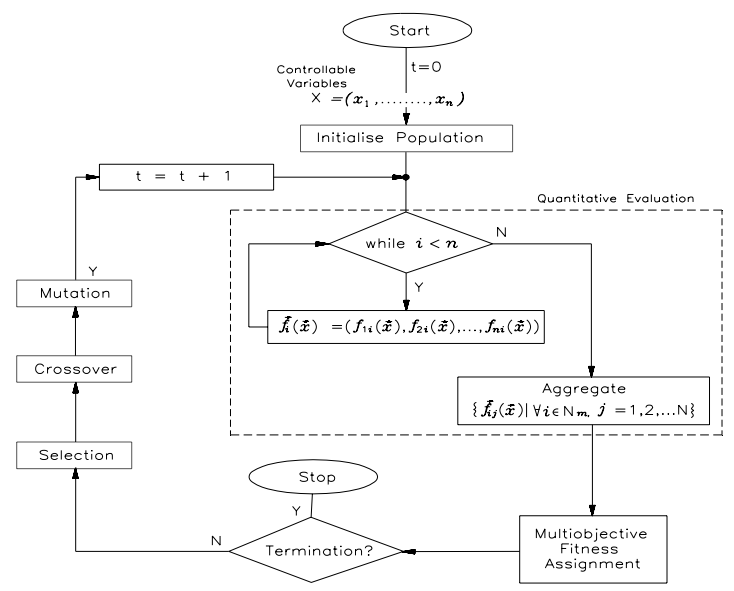

Fig. 1. NSGA-II for Sequential Optimisation Problems

The model is used in two steps to evaluate individual members of the population. The first step is a local evaluation of a sub-set of strings in the chromosomes. This represents the objective function values for each stage of the sequential process optimisation problem while the second part is a global evaluation. This aggregates the objective function values of all the stages using a suitable aggregation operator. The objective function value of the global evaluation represents the fitness of the chromosome. The dependency relationship is modelled by incorporating design variables (stock variables) from a previous pass $k=j-1$ into the objective function of pass $j$. This relationship is peculiar to those sequential processes, where the output stock of one stage serves as input stock into the deforming tool of the other stage (e.g. metal forming). This is expressed for a given stage $j$ by Equation 1, and the global evaluation is given by Equation 2 to illustrate this evaluation concept.

$$
\begin{gathered}
\vec{f}_{j}(\vec{x})=\left\{f_{i j}\left(x_{1 j}, x_{2 j}, \ldots . x_{m j}, x_{1 k}, x_{2 k}, \ldots . x_{r k}\right) \mid(j=1,2, \ldots n), k=j-1, r<m\right\} \\
\vec{F}_{i}^{k}(\vec{x})=\sum_{j=1}^{n} \vec{f}_{i j}^{k}(\vec{x}) \mid i=1,2, \ldots p
\end{gathered}
$$


where $i$ is the $i$ th objective, $p$ is the number of objectives, $m$ is the number of design variables at stage $j, k$ is the design variable from stage $j-1, r$ is the number of design variables from stage $j-1$ that is considered at stage $j$, and $n$ is the number of passes.

\subsection{Genetic String Representation Printing Area}

Figure 2 illustrates the string structure adopted for the sequential process optimisation problem. This coding procedure expresses all the design variables in binary code and combines them into a set of genetic strings that simulate the initial version of the design solution before conducting the evolutionary search. A genetic string is made up of sub-strings representing the number of stages, where the number of stages is assumed fixed. Since there is only one finishing stage, the genetic string has $n-1$ string segments for roughing and one segment for the finishing pass. Each of the substrings consists of product and process variables. Only controllable variables are represented in the genetic string and random values are chosen for these variables within the allowable range for each of the passes.

\begin{tabular}{|c|c|c|c|c|c|c|c|c|}
\hline$x_{11}$ & $x_{21}$ & $x_{m}$ & $x_{i_{2}}$ & $x_{22}$ & $x_{m 2}$ & $x_{n n}$ & $x_{2 n}$ & $x_{m n}$ \\
\hline 1000 & 1011. & 11110. & 1000. & $1011 \ldots$ & 0010 & $1001 \ldots$ & $0001 \ldots$ & $0110 \ldots$ \\
\hline
\end{tabular}

Fig. 2. String Structure of a Chromosome

\section{Case Study: Multi-pass Rolling System Optimisation}

The multi-pass rolling system is a high-speed continuous metal forming process where the metal from the reheating furnace (referred to as stock) is continuously deformed into the desired product geometry by passing through a series of rotating cylindrical rolls. The multi-pass rolling system design (RSD) optimisation attempts to locate optimal design solutions for individual passes of the rolling process including both design information such as the geometrical size of a roll and the operating conditions for the mills.

\subsection{Development of Multi-Pass Rolling Model}

A process model is required to evaluate the quality of each solution over the design space in order to conduct optimisation. Therefore, this section presents the development of a multi-pass rolling model. Figure 3 shows the process geometry addressed in this section. The overall breakdown sequence consists of a number of cascaded passes. The $i^{\text {th }}$ pass is denoted by $P_{i}$. Each pass is physically separated from its neighbours, and the output from the $P_{i-1}$ is provided to the $P_{i}$ as input. The breakdown 
sequence shows the oval stock turned through $90^{\circ}$ with its major axis in-line with the vertical axis of the round pass. This is repeated for all the subsequent passes. The model development is detailed as follows. A detailed description of the model is available in [10].
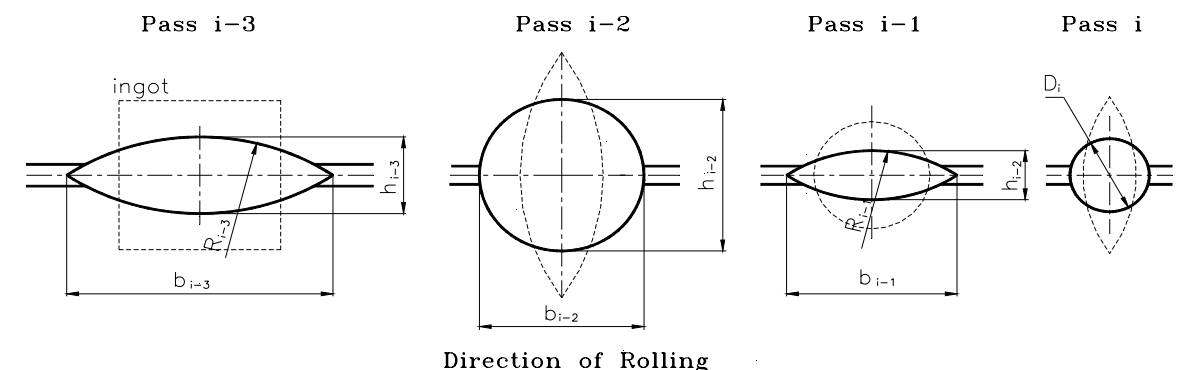

Fig. 3. Round-Oval-Round Breaking-Down Sequence

\section{Determination of Objective Function}

\section{Throughput}

Throughput $\left(T_{p}\right)$ is an important roll design objective that expresses the effect of mill productivity. It is expressed in terms of exit speed $(w)$ and final stock area $\left(A_{2}\right)$ :

$$
T_{p}=A_{2} w
$$

\section{Calculation of Pass Dimensions}

The pass variables for each oval pass are: $b_{j}$ and $h_{j}$; and round pass diameter, $D$. This assumes that the oval section is composed of two equal arcs of circle, its area is approximately [11]:

$$
A=\frac{2 h_{j} b_{j}}{2.85}
$$

The suffix ' $j$ ' $=(1,2)$ is for inlet and outlet variables respectively.

\section{Calculation of Roll Force}

One of the most important objectives in the scheduling is to provide an optimum rolling load $\left(P_{T}\right)$ required for deformation at each stand. Excessive loading in various passes can affect the productivity of the rolling process. The total deformation load cost function for total $N_{p}$ number of passes is defined as follows:

$$
P_{T}=\sum_{i=1}^{N_{p}}\left(P_{i}\right)
$$

Roll force formulation involves a complex interaction between the projected contact area between roll and stock and the mean resistance to deformation for which 
several authors have provided different expressions. Shinokura and Takai [12] proposed a simple equation for calculating effective roll radius, the projected contact area, the non-dimensional roll force and the torque arm coefficient expressed as a function of the geometry of the deformation zone. The formulation is adopted in this study since it is suitable for oval-to-oval passes. The equation is:

$$
P_{i}=\mathrm{Q}_{\mathrm{s}} \mathrm{A}_{\mathrm{p}}(2 \mathrm{k})
$$

where $Q_{s}$ is a multiplier, $A_{p}$ is the projected contact area and $2 k$ is the mean flow strength in the pass.

\section{Constraints for Validity Checks}

The industrial application imposes the introduction of some constraints related to the quality of the product, technical function and organisational considerations. These factors can be classified into mechanical design constraints and variable constraints. The variable constraints are the upper and lower limits of the variables.

Roll Load

The total roll force is limited to the mechanical design limits of the rolling mills imposed by the roll manufactures. Excessive roll load can cause roll breakage that can be detrimental to production efficiency. This constraint can be formulated as follows:

$$
g_{1}(\mathbf{x}) \equiv P_{i} \leq P_{\max }
$$

Related Process Functions

$\lambda_{\mathrm{T}}$ and $\lambda_{\mathrm{k}}$ are the total elongation and elongation at stage $k$, therefore:

$$
g_{2}(\mathbf{x}) \equiv \sum_{k=1}^{n} \lambda_{k}-\lambda_{T} \geq 0
$$

The following constraint is required to ensure the breakdown sequence is achieved with reduction taking place from pass to pass. For every pass $i$;

$$
\text { Inter-pass reduction } \quad g_{3}(\mathbf{x}) \equiv \lambda_{i}>1
$$

\section{Rod Size}

The overall rod size is a measure of product quality. This is allowed to vary within a given tolerance limit $K$. This is treated as a soft constraint to allow the possibility to explore designs within the specified boundaries. This is specified as:

$$
g_{4}(\mathbf{x}) \equiv K_{\min } \leq \sqrt{\left(D_{N p}-D\right)^{2}} \leq K_{\max }
$$

The breakdown sequence shows the oval stock turned through $90^{\circ}$ with its major axis in the vertical diagonal of the square pass. The square stock is provided as flat into the oval pass and the oval stock is turned through $90^{\circ}$ with its major axis in-line with the vertical axis of the round pass. Constraints on inter-pass sections can be shown as: 


$$
\begin{aligned}
& h_{3}(\mathbf{x}) \equiv b_{i}^{j}=h_{i-1}^{j+1} ; i=1,2 \ldots n ; j=1,2 \\
& h_{4}(\mathbf{x}) \equiv h_{i}^{j}=b_{i-1}^{j+1} ; i=1,2 \ldots n ; j=1,2
\end{aligned}
$$

\subsection{Multi-Pass Rolling System Optimisation}

This section presents an application of the novel framework (Section 2) for optimal shape design of a pass schedule in long product hot rolling of sections. The aim is to determine the geometry of each pass that would ensure the reduction of an ingot from a given section to a prescribed final section with respect to some cost objectives, while satisfying some constraints. The problem is solved using the proposed framework and the results are compared with work reported in the literature. GA computes the objective function for each string of the solution space so that the individual with the better fitness value is determined. Equation 13 gives a formal definition of the multi-objective RSD optimisation problem. It aims to minimise deformation load and maximise rolling throughput, subject to given constraints for a four pass oval to round design. The deformation load is a cost objective while throughput is a function of mill productivity. Excessive deformation load results in excessive roll wear and hence overall production cost. Both objectives are assumed conflicting in nature since metal compression by the cylindrical rolls encourages metal flow in the direction of rolling, increasing the metal deformation load, also increasing the metal flow.

\section{Equation 13:}

Minimise Total Deformation Load (5) $f_{1}(\mathbf{x}) \equiv P_{T}(x)$

Maximise Throughput (3)

$$
f_{2}(\mathbf{x}) \equiv T_{p}(x)
$$

Subject to:

Roll Load (7)

$$
g_{1}(\mathbf{x}) \equiv P_{i} \leq P_{\max }
$$

Total elongation (8)

$$
g_{2}(\mathbf{x}) \equiv \sum_{k=1}^{n} \lambda_{k}-\lambda_{T} \geq 0
$$

Inter-pass reduction (9)

$$
g_{3}(\mathbf{x}) \equiv \lambda_{i}>1
$$

Rod size (10)

$$
g_{4}(\mathbf{x}) \equiv K_{\min } \leq \sqrt{\left(D_{N p}-D\right)^{2}} \leq K_{\max }
$$

Inter-pass sections (11)

$$
h_{3}(\mathbf{x}) \equiv b_{i}^{j}=h_{i-1}^{j+1} ; i=1,2 \ldots n ; j=1,2
$$




\section{Experimental Details}

The NSGA-II based algorithm solves a multi-pass roll design problem to minimise deformation load while maximising the mill throughput. Experiments were carried out using the proposed framework for the two objectives to illustrate how the algorithm deals with the multi-objective multi-pass search space problem. The following additional parameters were set for the experiment. $P_{\max }=750 \mathrm{KN}$ per stand, final rod diameter $(D)=20.1 \pm 0.25 \mathrm{~mm}$, where $k_{\min }$ is set as $19.85 \mathrm{~mm}$ and $k_{\max }$ is set as $20.35 \mathrm{~mm}$, and the tolerance range was estimated based on design experience.

Results were obtained using the process model developed in Section 3.1. The performances of the solution algorithm for different values of crossover and mutation probabilities were first investigated. Ten independent GA runs were performed in each case using a different random initial population. The crowded tournament selector operator was used to select new offspring. Tests were carried out with the following parameters: population of size 500 for 2000 generation with a 3-point crossover probability of 0.8 and a mutation probability of 0.03 , and tournament selection with size 3. The 3-point crossover was chosen to ensure more effective transfer of genetic material during reproduction since the total chromosome length is 225. These results form the typical set obtained from 10 runs with different random number seeds. Six out of ten runs obtained similar results. A random search of 10000 points is also conducted in order to get an indication of the search space. This will be used to identify the likely presence of a Pareto front in the design problem. Details of results are discussed as follows.

\section{Discussion of Results}

The results obtained from the NSGA-II algorithm are compared to empirical results published in the literature [11]. Results shown in Figure 4 were obtained for the NSGA-II parameters outlined above. Comparison of the random search space and the results achieved from the algorithm confirms that the solution algorithm has been able to converge to the Pareto front. Since the search space is not known in absolute terms, the results reported in Figure 4 have converged to the near optimal Pareto front locating a reasonable spread of multiple optimal solutions. The presence of a Pareto front also confirms the conflicting relationship between deformation load and throughput. The empirical design point obtained from the literature was superimposed in the search space and compared with the near optimal solutions. Since this point does not lie on the identified near optimal Pareto front, it is clear that the solutions obtained are superior to the empirical based solution. 


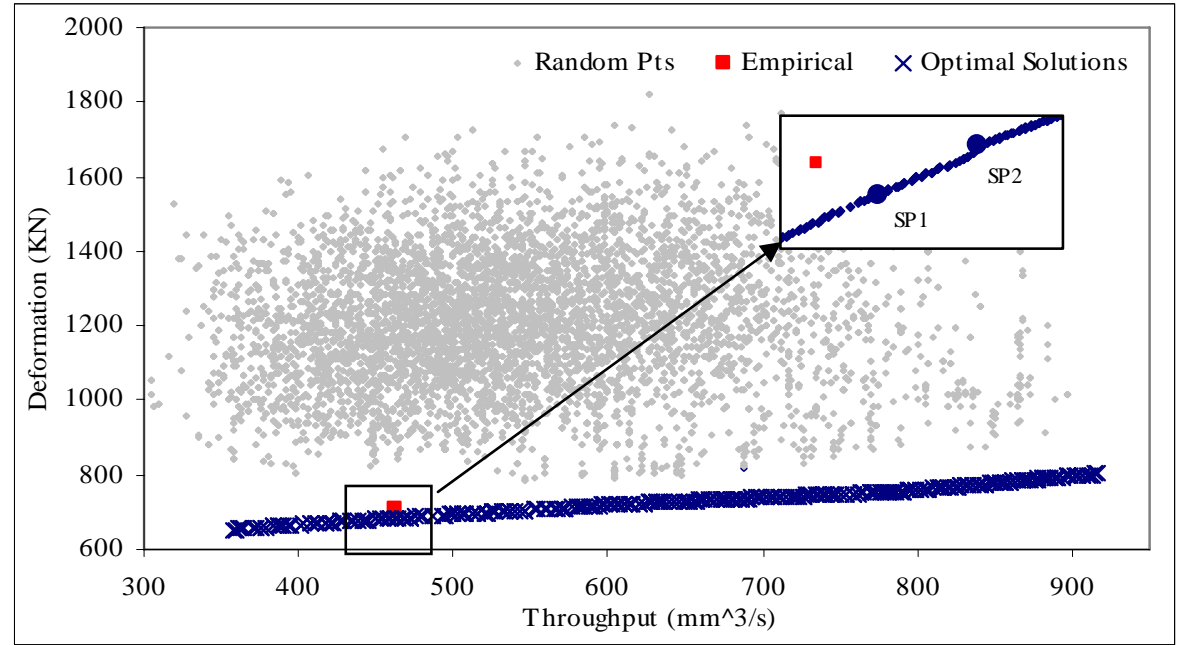

Fig. 4. Pareto Front for Four Pass Rolling Process

SP1 (see insert in Figure 4) demonstrates a classic example of how this algorithmic-based approach can locate better solutions compared to the single point methods. Since the deformation load is lower and the throughput is higher than the empirical solution, the NSGA-II based solution is better than the empirical based solution for both objectives. This implies that the proposed approach is capable of identifying solutions with higher outputs at lower deformation loads than the empirical based solution. Two solution points SP1 and SP2 are selected (see insert in Figure 4) and compared with the empirical solution in terms of throughput, deformation load, elongation coefficient and the deformed sections. Results obtained from the proposed approach not only identify good solutions, but also provide insight into the complex behaviour of the design problem.

The optimisation framework is limited to process optimisation problems with sequential relationships between consecutive stages. Also, the case study only considers a four pass rolling design, in practice there can be many more passes required for rod rolling (usually up to 25 passes). The scalability of the framework is not tested in this research. It is also observed that aggregation of the contributions from different passes may even out fluctuations caused by many of the underlying unknown factors, this can lead to deception problem for the GA. In future it would be useful to study the nature of behaviour between the different stages during the GA search. Such information could be valuable in understanding the convergence behaviour of the search space, which could result in improved GA based optimisation algorithms. 


\section{Conclusion}

This paper has proposed a novel GA-based optimisation framework for dealing with real-life sequential process optimisation problems. The proposed framework has been validated using a real-life case study involving the multi-objective optimisation of a multi-pass rolling system design. The near optimal results obtained prove the effectiveness of the proposed framework.

\section{References}

1. Simon, H., 1972, Theories of Bounded Rationality, in Decision and Organization, R. Radner, Editor, North Holland.

2. Kobayashi, S., 1987, Process design in Metal Formng by the Finite Element Method. Advanced Technology of Plasticity, 11: 1213-1219.

3. Hwang, S.M. and Kobayashi, S., 1984, Preform design in plane-strain rolling by the finite element method. International Journal of Machine Tool Design and Research, 24: 253-266.

4. Joun, M.S. and Hwang S. M., 1993, Optimal Process Design in Steady-State Metal Forming by Finite Element Method-II. Application to Die Profile Design in Extrusion. International Journal of Machine and Tool Manufacture, 33(1): 63-70.

5. Deb, K., 2001, Multi-objective Optimization Using Evolutionary Algorithms. John Wiley and Sons Ltd.

6. Chakraborti, N. and Kumar, A., 2003, The Optimal Scheduling of a Reversing Strip Mill: Studies Using Multi-population Genetic Algorithms and Differential Evolution. Journal of Material Processing Technology. Submitted for publication.

7. Goldberg, D.E., 1989, Genetic Algorithm in Search, Optimization and Machine Learning. Addison Wesley, Massachusetts.

8. Roy, S., Ghosh, S., and Shivpuri, R., 1996, Optimal Design of Process Variables in MultiPass Wire Drawing by Genetic Algorithms. Journal of Manufacturing Science and Engineering, 118-124.

9. Hwang, S.M. and Chung, J.S., 1997, Application of genetic algorithm to optimal design of the die shape in Extrusion. Journal of Materials Processing Technology, 72: 69-77.

10.Oduguwa, V., 2003, Rolling System Design Optimisation using Soft Computing Techniques, EngD Thesis, Cranfield University, UK.

11.Wusatowski, Z., 1969, Fundamentals of rolling, Pergamon.

12.Shinokura, T. and Takai, K., 1982, A new method for calculating spread in rod rolling. Journal of Application in Metalworking, 2: 147-160. 